\title{
Original Prevalence of high anti tissue transglutaminase (TTG) antibody levels among 'at high risk' population in north India
}

\author{
Ajit Sood ${ }^{1}$, Parneet Wander ${ }^{1}$, Kirandeep Kaur ${ }^{2}$, Ramit \\ Mahajan $^{2}$, Kriti Sood ${ }^{3}$, Vandana Midha ${ }^{4}$
}

ABSTRACT

${ }^{1}$ Department of Gastroenterology,

${ }^{2}$ Department of Pharmacology,

${ }^{3}$ Department of Paediatrics,

${ }^{4}$ Department of Medicine, Dayanand

Medical College and Hospital,

Ludhiana, Punjab.

Correspondence: Ajit Sood

Email: ajitsood10@gmail.com
Background: Although prevalence of celiac disease (CD) is known from the western population, there is a lack of epidemiological data from India. This study aimed at determining the prevalence of $\mathrm{CD}$ among at high risk population in India.

Study Design: Patients considered to be at high risk for CD i.e. those presenting with iron deficiency anemia (IDA) or type 1 diabetes mellitus (T1DM) or infertility or idiopathic generalized epilepsy (IGE) or the firstdegree relatives of patients of $\mathrm{CD}$ were enrolled in the study. The study was conducted in a tertiary care of hospital with enrollment period from September 2012 to August 2013. They were screened for CD and anti tissue transglutaminase (tTG) antibody levels; and those who tested positive and consented, underwent endoscopic duodenal biopsy for confirmation.

Results: Prevalence of high anti-tTG was found in $27.3 \%$ of IDA patients, $4.3 \%$ among patients with T1DM, $12.8 \%$ of infertile female patients, $3 \%$ of the patients with IGE and $23.4 \%$ of the first degree relatives. Prevalence of villous atrophy was found in $4 \%$ of patients with IDA, $4.3 \%$ among those with T1DM, 1.8\% among infertile female patients and 3\% among patients with IGE. None of the first degree relatives consented for intestinal biopsy. Conclusion: Prevalence of $\mathrm{CD}$ was high among patients presenting with IDA, T1DM and IGE. Prevalence couldn't be calculated in first degree relatives because of non-consenting patients. Better awareness among both patients and physicians is required in our country.

KEYWORDS: Celiac disease; prevalence; high risk; India. 


\section{Introduction}

The changing epidemiological profile of celiac disease (CD) shows an increase in prevalence rate and existence of distinct adult and pediatric presentation of the disease. ${ }^{1,2}$ World over, prevalence rate of approximately $1 \%$, ranging from $0.71-1.25 \%$ with female preponderance in early age group has been reported. ${ }^{1,3}$ Contrary to earlier thoughts of low prevalence rates among Indian population, recent evidence shows an increasing prevalence even in India, especially North India. ${ }^{4-6}$ However, we have limited epidemiological data from India and the currently known pooled prevalence rate is approximately $0.6-1 \% .^{4-6}$ The increased prevalence across different geographical areas might be because of advancement in diagnostic aspects and change in environmental risk factors. ${ }^{2}$ The availability of highly sensitive and specific tests like serologic evaluation are helping in easy and accurate screening for CD. However, CD remains under-reported and undiagnosed among a large section of persons. ${ }^{3,7}$

Celiac Disease is known to be associated with presence of certain autoimmune disease conditions. Also, a higher prevalence rate of CD has been reported among certain groups of patients, often referred as 'at high risk'. ${ }^{3}$ Some of the known associations between autoimmune diseases and $\mathrm{CD}$ are, like, type 1 diabetes mellitus (T1DM), thyroiditis and dermatitis herpetiformis. Other associations are with extra-intestinal manifestations like osteoporosis, iron deficiency anemia (IDA), cryptogenic hypertransaminasia, miscarriages and neurologic problems. ${ }^{2,3,7}$ Thus, patients having T1DM or IDA or infertility or idiopathic seizures or with positive family history of $\mathrm{CD}$ are at a higher risk of being diagnosed with $\mathrm{CD}^{3}$

During the last half century, various epidemiological and clinical studies of CD have shown an increased risk of infertility, intrauterine growth retardation (IUGR), low birth weight babies, preterm birth, spontaneous abortion, delayed puberty and precocious menopause among female patients. ${ }^{8}$ Besides these studies, contradictory results are also available for no difference in prevalence of infertility among the $\mathrm{CD}$ patients and general population. Prevalence of CD has been reported to be like general population $(<1 \%)$ to as high as $4-8 \%$ among infertile females. ${ }^{89}$ Similarly, European studies have also shown varied results ranging from normal fertility to a prevalence rate of $2.5-3.5 \%$ among women with CD. ${ }^{10,11}$ On the other hand, an Indian study has shown a sero-prevalence of $6.7 \%$ among patients with recurrent abortion and $5.65 \%$ among patients with unexplained infertility. ${ }^{12}$

The second at high risk sub-group includes patients with T1DM, as prevalence ranging from 3-16\% of $\mathrm{CD}$ has been reported from this group. ${ }^{13}$ There is a patho-genetic link between the two conditions as patients with co-occurrence of these two conditions have HLA class II risk genotypes. ${ }^{14}$ Approximately $30 \%$ of patients homozygous for HLADQ2 show positive results with presence of anti-tissue transglutaminase (anti-tTG) IgA antibodies. ${ }^{14}$ Patients presenting with IDA, or with IGE or the first degree relatives of $\mathrm{CD}$ patients form other high risk sub-groups. ${ }^{15-21}$ As limited number of studies have evaluated this association, a wide range (1.6-38\%) of prevalence of $\mathrm{CD}$ has been reported among the first degree relatives of $\mathrm{CD}$ patients. ${ }^{22}$ Till now, Indian data from North India is of $8.2 \%$ and $10.9 \%$ among the first degree relatives of $\mathrm{CD}$ patients. ${ }^{23,24}$ There is a need to have more data from a wider base.

Amidst this scenario, we continued our observational epidemiological study of evaluating the prevalence of CD among at high risk patients. We aimed to assess the prevalence of subclinical CD by means of serology and intestinal biopsy examination among patients with a history of infertility or T1DM or IDA or idiopathic generalized seizures or the first degree relatives of patients with $\mathrm{CD}$.

\section{Methods}

This was a prospective observational study conducted in the Out Patients Department of Dayanand Medical College, Ludhiana.

\section{Target population}

During a period of one year (September 2012 to August 2013), patients presenting with (T1DM); (IDA); 
infertility (female patients); or idiopathic seizures in the general medicine OPD, Infertility clinic, Epilepsy Clinic respectively were evaluated for celiac disease. Additionally, first degree relatives of patients diagnosed with CD presenting in the Gastroenterology OPD were also included.

All patients/subjects who gave informed consent for participation in the study with age $\geq 18$ years and diagnosed with the following criteria were included in the study.

\section{Iron deficiency anemia}

Patients with transferrin saturation $<10 \%$ and serum ferritin $<10 \mu \mathrm{g} / 1$ and microcytic/hypochromic anemia on peripheral blood film were diagnosed as having IDA. ${ }^{25}$ Complete history was taken to rule out any cause of IDA i.e. hookworm infestation, history of excessive blood loss, pregnancy, poor dietary habits.

\section{$T 1 D M$}

Diagnosis of T1DM was established by the presence of any of the following two criteria: (a) age of onset of diabetes mellitus less than 35 years; (b) Interval of $<3$ years between onset of DM and requirement of insulin; (c) body mass index $<25 \mathrm{~kg} / \mathrm{m}^{2}$ and $27 \mathrm{~kg} / \mathrm{m}^{2}$ for females and males respectively; (d) Presence of antibodies to islet cells.

\section{Infertility}

Female patients were diagnosed as infertile if there was a failure to achieve a clinical pregnancy after 12 months or more of regular unprotected sexual intercourse. ${ }^{26}$ The spouses were also investigated for infertility. Detailed history was taken and investigations were done to rule out any underlying cause of infertility. Thyroid hormone (T3 and T4) and TSH levels, FSH, LH, prolactin, progesterone and estrogen levels were measured. Investigations like hysterosalphingography, endometrial biopsy, and diagnostic laparoscopy were also conducted.

\section{Idiopathic generalized epilepsies (IGE)}

Complete history was taken to rule out any underlying cause of seizures. The patients were diagnosed according to International League Against Epilepsy definition, which states that IGE are forms of generalized epilepsies in which all seizures are initially generalized (absences, myoclonic jerks and generalized tonic clonic seizures), with an EEG expression that is a generalized bilateral, synchronous, symmetrical discharge (such as is described in the seizure classification of the corresponding type). ${ }^{27}$

\section{First degree relatives}

During the above mentioned period, first degree relatives (parents, siblings and children) of index cases of CD presenting in Gastroenterology $\mathrm{OPD}, \mathrm{DMCH}$, were screened.

All consenting patients were inquired for gastrointestinal signs and symptoms. Complete personal and family history was taken. All consenting subjects underwent quantitative measurement of anti-tTG IgA (Bio-RAD Autoimmune EIA) levels. The reference range for this assay was $0-10 \mathrm{U} / \mathrm{ml}$. Patients having levels $>10$ $\mathrm{U} / \mathrm{mL}$ were considered to be serologically positive for $\mathrm{CD}$. These positive cases were then offered endoscopic intestinal biopsies for confirmation of $\mathrm{CD}$. The diagnosis of $\mathrm{CD}$ was based on modified European Society of Pediatric Gastroenterology, Hepatology and Nutrition (ESPGHAN) criteria. ${ }^{28}$ This study was approved by the Institutional Ethics Committee.

\section{Results}

\section{Iron deficiency anemia}

A total of 99 cases of IDA were diagnosed and 27 had increased anti-tTG levels (serologically 27.3\% tested positive) and only seven consented for biopsy and three had normal and four patients had villous atrophy showing a prevalence of $4 \%$ (Table 1 ).

\section{Type $1 D M$}

Out of the 94 screened patients, four had elevated levels of anti-tTG (serologically $4.3 \%$ tested positive), these four 
consented for intestinal biopsy and all these patients had villous atrophy on intestinal biopsy examination, showing a prevalence of $4.3 \%$ (Table 1).

\section{Infertility}

A total of 218 patients reported to the Infertility clinic during this period and 28 had raised anti-tTG levels (serologically $12.8 \%$ tested positive); further only six patients consented for biopsy and four had villous atrophy and two had normal presentation, showing a prevalence of $1.83 \%$ (Table 1 ).

\section{Idiopathic generalized epilepsy}

Out of 168 consenting patients for screening, five had high anti-tTG levels (2.97\%) and these five further consented for biopsy and all of them had findings suggestive of CD (stage IIIa); thus a prevalence of $2.97 \%$ (Table 1).

\section{First degree relatives}

A total of 42 families with 128 members were included and out of these, 111 members consented for screening; 26 first degree relatives had high anti-tTG levels (serologically $23.4 \%$ tested positive). None of the patients consented for biopsy, thus no confirmation of CD could be made (Table 1).

\section{Discussion}

This observational study of one year duration shows a prevalence of $4 \%, 4.3 \%, 1.8 \%$ and $3 \%$ of biopsy confirmed CD among patients presenting with IDA, T1DM, infertility and idiopathic generalized seizures, respectively. We had intended to study prevalence of CD among five high risk groups, but, because of the nonconsent by the patients for intestinal biopsy, we couldn't establish the prevalence among these groups accurately especially among the infertile female patients and first degree relatives of $C D$ patients. This series is a part of our ongoing effort to report the prevalence rate of $\mathrm{CD}$ among North Indian patients belonging to the at high risk group.

Although our study shows a nil prevalence of biopsy confirmed $\mathrm{CD}$ among infertile females, this data is insufficient to come to the conclusion of non-existence of $\mathrm{CD}$ in this high risk group. The non-consenting patients hampered our evaluation of prevalence of CD. Out of 28 (12.8\%) patients with elevated anti-tTG levels, only six consented for further evaluation by intestinal biopsy. So, diagnosis couldn't be established in the rest of the target group included in the study. Variable results are available from literature with respect to prevalence of CD among infertile patients. Here, we cannot compare our results as majority of the patients did not give consent for biopsy. Earlier reports by Meloni and Collin et al. have shown prevalence rate of $4.1 \%$ and $3 \%$ among infertile women. ${ }^{11,29}$ Later, Pellicano et al. reported prevalence of CD to be 2.5-3.5\% among women with unexplained infertility, which was higher, although not significantly, than control population. ${ }^{10}$ A study of infertile Brazilian women has reported the prevalence of $10.3 \%$ among patients with $\mathrm{CD}$ and unexplained infertility. ${ }^{30}$ Also, results of a metaanalysis shows that patients with unexplained infertility, recurrent miscarriage or IUGR have a significantly higher risk of $\mathrm{CD}$ than the general population. ${ }^{31}$ On the other

Table 1: Results

\begin{tabular}{l|c|c|c|c} 
High risk group & $\begin{array}{l}\text { Total patients } \\
\text { screened }\end{array}$ & $\begin{array}{l}\text { Patients tested } \\
\text { positive with high } \\
\text { anti-t TG levels }\end{array}$ & $\begin{array}{l}\text { Patients consented for } \\
\text { biopsy }\end{array}$ & $\begin{array}{l}\text { Patients tested positive } \\
\text { on biopsy }\end{array}$ \\
\hline First degree relatives & 111 & 26 & 07 & None \\
\hline IDA & 99 & 27 & 04 & 04 \\
\hline Type 1 DM & 94 & 04 & 06 & 04 \\
\hline Infertility & 218 & 28 & 05 & 05 \\
\hline $\begin{array}{l}\text { Idiopathic Generalized } \\
\text { Epilepsy }\end{array}$ & 168 & 05 & &
\end{tabular}


hand, Jackson et al. has mentioned a prevalence of $0.8 \%$ among infertile patients which is similar to the general population. ${ }^{9}$ With respect to Indian population, Kumar et al. have shown the sero-prevalence of anti-tTG antibodies to be $6.7 \%, 5.7 \%, 5.7 \%, 9.3 \%$ and $1.3 \%$ in the groups with recurrent abortion, stillbirth, infertility, intrauterine growth restriction, and in the control group respectively. Also, the rates of previous preterm births, low-birth-weight infants, and cesarean section were higher in seropositive women compared with seronegative subjects. ${ }^{12}$ The possible explanation for the increased occurrence of infertility among CD patients is the malabsorption of folic acid and other nutrients and the detrimental effects of anti-tTG antibodies on endometrial angiogenesis. ${ }^{10}$

The second high risk group included patients presenting with IDA as a clinical manifestation. Recent study from Kashmir reports prevalence of CD among $8 \%$ (both serological and biopsy confirmed) of patients with obscure IDA and gluten free diet (GFD) improved anemia among this group. ${ }^{32}$ Also, the lower hemoglobin concentration was correlated with higher Marsh Grade $(\mathrm{p}=0.001) .{ }^{32}$ However, our study reports serological and biopsy confirmed prevalence of $27.3 \%$ and $4 \%$ respectively. Ucardag et al. have shown both serological and biopsy confirmed prevalence of $7.8 \%$ among Turkish population with IDA of obscure origin. ${ }^{33}$ Again, the unconsenting patients intervened in our aim of evaluating the prevalence of biopsy confirmed $\mathrm{CD}$ among patients presenting with IDA.

On the similar lines, various epidemiological studies have shown a high prevalence of $C D$ among T1DM patients. ${ }^{15,17,34}$ The association of T1DM and $\mathrm{CD}$ has been explained by co-existence of HLA-DQ2/

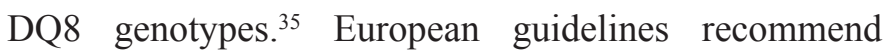
screening for these genotypes as CD is unlikely if both haplotypes are negative. ${ }^{28}$ In India, prevalence studies are now being conducted for understanding this association. Six years long study has shown a prevalence of $11.1 \%$ among patients with T1DM and $90.5 \%$ were diagnosed on screening. ${ }^{17}$ Prevalence of $\mathrm{CD}$ has been reported among $15.5 \%$ children with T1DM by Joshi et al. The biopsy confirmed CD was seen in 7\% patients and one third of the patients were symptomatic and presented with intestinal symptoms, anemia, rickets and short stature. ${ }^{15}$ Our prevalence rates are less as compared to Asian studies. ${ }^{34}$ Although association of CD with infertility, IDA and T1DM has been studied extensively, less information is available about the prevalence of $\mathrm{CD}$ among patients presenting with neurological signs. Prevalence ranging from $0.8 \%$ to $9.1 \%$ has been reported world over. ${ }^{36}$ Prevalence rate of $2.9 \%$ among our subjects lies within this range only.

The last sub-group of our study was of first degree relatives of $\mathrm{CD}$ patients. In an earlier report, we had mentioned that $\mathrm{CD}$ is common among siblings of patients with CD in North India and it may be symptomatic. Out of 63 siblings, 22\% had biopsy confirmed $\mathrm{CD}$, including eight patients who had no symptoms suggestive of CD. ${ }^{18}$ This relation has also been investigated and established world over. ${ }^{37}$ Reports from Italy have shown a prevalence of $23.8 \%$ and $20.1 \%$ among siblings and family members respectively. ${ }^{37}$ Mishra et al. found that $10.9 \%$ of first degree relatives had CD and $87 \%$ had HLA-DQ2 or DQ8 haplotype. ${ }^{24}$ Grover et al. and Srivastava et al. have reported a prevalence of $8.2 \%$ and $4.4 \%$ respectively among first degree relatives. ${ }^{23,38}$ Here, we couldn't calculate the prevalence of $\mathrm{CD}$ as patients didn't give consent for biopsy, thus leading to an incomplete picture.

The analysis of our results draws our attention towards the limiting factors in our screening methods. The lack of awareness and conviction among both the health care providers and the patients decreased the number of patients consenting for the endoscopic biopsy for confirmation of the diagnosis. Although, the serological tests are highly sensitive and specific, yet the diagnosis of $\mathrm{CD}$ is established by intestinal mucosal biopsy when the patient is on gluten containing diet. ${ }^{3,7,34,39}$ Among mixed population i.e. including both adults and children, anti-tTG sensitivity and specificity have been reported to be $93.7 \%$ and $95.4 \%$ respectively. ${ }^{3,34}$ The sensitivity and specificity of serologic tests also depends upon the prevalence rate in the geographical area, the grade of histological involvement and the anti-tTG values., ${ }^{3,34}$ In our study, had we got consent for biopsy examination by majority of the patients, we would have been able to accurately establish the prevalence rate of CD among at high risk population in this geographical area. 
As it is vital to fill the void of Indian epidemiological data, we tried to collect data from a tertiary institute of North India. In the current scenario, it is important that healthcare professionals of different specialties should be aware of the persons grouped as at high risk for prevalence of CD. If the treating physician is convinced to confirm the diagnosis of $\mathrm{CD}$ by intestinal biopsy among high risk population, it will definitely increase the number of persons opting for this investigation. As European countries are discussing the inclusion of HLA genotyping in screening of T1DM patients for $\mathrm{CD}$, maybe it is time for us to consider serological screening in our healthcare system as well. ${ }^{35}$ Presently, there is no recommendation for screening by anti-tTG levels among high risk patients. There is a need to develop a consensus and policy on the requirement of screening of CD among at high risk population.

Other limiting factors of this study include the short duration of study and lack of follow-up as it was a one point study. The results of our analysis are comparable to those found in other Indian studies, but all of these, including ours are single hospital based studies limiting their clinical utilization and implication. The available evidence shows that $\mathrm{CD}$ should be considered as a possible cause of clinical manifestations among at high risk patients.

\section{Conclusion}

Screening of patients presenting with IDA, IGE and T1DM led to identification of patients with biopsy confirmed CD. There is a need to generate more evidence about prevalence of $\mathrm{CD}$ among at high risk group, which can help in formulating guidelines for requirement of screening among these patients.

\section{References}

1. Vivas S, Vaquero L, Rodríguez-Martín L, Caminero A. Age-related differences in celiac disease: Specific characteristics of adult presentation. World J Gastrointest Pharmacol Ther. 2015;6(4):207-12.

2. Volta U, Caio G, Stanghellini V, De Giorgio R. The changing clinical profile of celiac disease: a 15-year experience (1998-2012) in an Italian referral center. BMC Gastroenterol. 2014;14:194.

3. Rostom A, Murray JA, Kagnoff MF. American Gastroenterological Association (AGA) Institute technical review on the diagnosis and management of celiac disease. Gastroenterology. 2006;131(6):1981-2002.

4. Yachha SK, Poddar U. Celiac disease in India. Indian $J$ Gastroenterol. 2007;26(5):230-7.

5. Bhattacharya M, Dubey AP, Mathur NB. Prevalence of celiac disease in north Indian children. Indian Pediatr. 2009;46(5):415

6. Makharia GK, Verma AK, Amarchand R, et al. Prevalence of celiac disease in the northern part of India: a community based study. J Gastroenterol Hepatol. 2011;26(5):894-900.

7. Ludvigsson JF, Leffler DA, Bai JC, et al. The Oslo definitions for coeliac disease and related terms. Gut. 2013;62(1):43-52.

8. Fortunato F, Martinelli D, Prato R, Pedalino B. Results from ad hoc and routinely collected data among celiac women with infertility or pregnancy related disorders: Italy, 20012011. Scientific World Journal. 2014;2014:614269.

9. Jackson JE, Rosen M, McLean T et al. Prevalence of celiac disease in a cohort of women with unexplained infertility. Fertil Steril. 2008;89(4):1002-4

10. Pellicano R, Astegiano M, Bruno M, Fagoonee S, Rizzetto M. Women and celiac disease: association with unexplained infertility. Minerva Med. 2007;98(3):217-9.

11. Meloni GF, Dessole S, Vargiu N, Tomasi PA, Musumeci S. The prevalence of coeliac disease in infertility. Hum Reprod. 1999;14(11):2759-61.

12. Kumar A, Meena M, Begum N, et al. Latent celiac disease in reproductive performance of women. Fertil Steril. 2011;95(3):922-7

13. Pham-Short A, Donaghue KC, Ambler G et al. Screening for Celiac Disease in Type 1 Diabetes: A Systematic Review. Pediatrics. 2015;136(1):e170-6.

14. Abenavoli L, Delibasic M, Peta V et al. Nutritional profile of adult patients with celiac disease. Eur Rev Med Pharmacol Sci. 2015;19(22):4285-92

15. Joshi R, Madvariya M. Prevalence and clinical profile of celiac disease in children with type 1 diabetes mellitus. Indian J Endocrinol Metab. 2015;19(6):797-803.

16. Cannizzaro R, Da Ponte A, Tabuso M et al. Improving detection of celiac disease patients: a prospective study in iron-deficient blood donors without anemia in north Italy. Eur J Gastroenterol Hepatol. 2014;26(7):721-4

17. Bhadada SK, Kochhar R, Bhansali A et al. Prevalence and 
clinical profile of celiac disease in type 1 diabetes mellitus in north India. J Gastroenterol Hepatol. 2011;26(2):37881.

18. Gautam A, Jain BK, Midha V, Sood A, Sood N. Prevalence of celiac disease among siblings of celiac disease patients. Indian J Gastroenterol. 2006;25(5):233-5.

19. Mavroudi A, Xinias I, Papastavrou Tet al. Increased prevalence of silent celiac disease among Greek epileptic children. Pediatr Neurol. 2007;36(3):165-9.

20. Pratesi R, Gandolfi L, Martins RC et al. Is the prevalence of celiac disease increased among epileptic patients? Arq Neuropsiquiatr. 2003;61(2B):330-4.

21. Ranua J, Luoma K, Auvinen Aet al. Celiac disease-related antibodies in an epilepsy cohort and matched reference population. Epilepsy Behav. 2005;6(3):388-92.

22. Singh P, Arora S, Singh A, Strand TA, Makharia GK. Prevalence of Celiac disease in Asia: A systematic review and meta-analysis. $J$ Gastroenterol Hepatol. 2016;31(6):1095-101.

23. Grover R, Puri AS, Aggarwal N, Sakhuja P. Familial prevalence among first-degree relatives of celiac disease in North India. Dig Liver Dis. 2007;39(10):903-7.

24. Mishra A, Prakash S, Kaur Get al. Prevalence of celiac disease among first-degree relatives of Indian celiac disease patients. Dig Liver Dis. 2016;48(3):255-9.

25. Kaushansky K, Kipps TJ. Hematopoietic Agents: Growth Factors, Minerals and Vitamines In: Laurance L. Brunton, Goodman \& Gilman's The pharmacological Basis of Therapeutics, 12th edition, New York:McGraw Hill:2011.p.1067-99

26. WHO. http://www.who.int/reproductivehealth/topics/ infertility/definitions/en/ (Last accessed on 3rd April, 2016)

27. Panayiotopoulos CP. The Epilepsies: Seizures, Syndromes and Management. Oxfordshire (UK): Bladon Medical Publishing; 2005. Chapter 10, Idiopathic Generalised Epilepsies. Available from: http://www.ncbi.nlm.nih.gov/ books/NBK2608/

28. Husby S, Koletzko S, Korponay-Szabó IRet al; ESPGHAN Working Group on Coeliac Disease Diagnosis; ESPGHAN Gastroenterology Committee; European Society for Pediatric Gastroenterology, Hepatology, and Nutrition.
European Society for Pediatric Gastroenterology, Hepatology, and Nutrition guidelines for the diagnosis of coeliac disease. $J$ Pediatr Gastroenterol Nutr. 2012;54(1):136-60.

29. Collin P, Vilska S, Heinonen PK, Hällström O, Pikkarainen P. Infertility and coeliac disease. Gut. 1996;39(3):382-4

30. Machado AP, Silva LR, Zausner B et al. Undiagnosed celiac disease in women with infertility. $J$ Reprod Med. 2013;58(1-2):61-6.

31. Tersigni $\mathrm{C}$, Castellani $\mathrm{R}$, de Waure $\mathrm{C}$ et al. Celiac disease and reproductive disorders: meta-analysis of epidemiologic associations and potential pathogenic mechanisms. Hum Reprod Update. 2014;20(4):582-93

32. Javid G, Lone SN, Shoukat Aet al. Prevalence of celiac disease in adult patients with iron-deficiency anemia of obscure origin in Kashmir (India). Indian J Gastroenterol. 2015;34(4):314-9.

33. Uçardağ D, Güliter S, Ceneli $\mathrm{O}$ et al. Celiac disease prevalence in patients with iron deficiency anemia of obscure origin. Turk J Gastroenterol. 2009;20(4):266-70.

34. Al-Hussaini A, Sulaiman N, Al-Zahrani M, Alenizi A, El Haj I. High prevalence of celiac disease among Saudi children with type 1 diabetes: a prospective cross-sectional study. BMC Gastroenterol. 2012;12:180

35. Mitchell RT, Sun A, Mayo A et al. Coeliac screening in a Scottish cohort of children with type 1 diabetes mellitus: is DQ typing the way forward? 2016;101(3):230-3.

36. Vieira C, Jatobá I, Matos M, Diniz-Santos D, Silva LR. Prevalence of celiac disease in children with epilepsy. Arq Gastroenterol. 2013;50(4):290-6.

37. Bardella MT, Elli L, Velio P et al. Silent celiac disease is frequent in the siblings of newly diagnosed celiac patients. Digestion. 2007;75(4):182-7.

38. Srivastava A, Yachha SK, Mathias A et al. Prevalence, human leukocyte antigen typing and strategy for screening among Asian first-degree relatives of children with celiac disease. J Gastroenterol Hepatol. 2010;25(2):319-24.

39. Maglione MA, Okunogbe A, Ewing Bet al. Diagnosis of Celiac Disease [Internet]. Rockville (MD): Agency for Healthcare Research and Quality (US); 2016 Jan. Available From http://www.ncbi.nlm.nih.gov/books/NBK344454/ 